\title{
Weak anisotropic impurity scattering in unconventional superconductors
}

\author{
Grzegorz Haran't and A. D. S. Nagi \\ Department of Physics, University of Waterloo, Waterloo, Ontario, Canada, N2L 3G1
}

(July 24, 1996)

\begin{abstract}
The effect of weak anisotropic (momentum-dependent) impurity scattering in unconventional superconductors has been investigated. It is shown that the anisotropic scattering can lead either to a small reduction or a small enhancement of the isotropic pair-breaking effect. The influence of the anisotropy of the scattering potential becomes significant for the order parameters with large Fermi surface average values. In that case an unexpected enhancement (up to $\sim 10 \%$ ) of the critical temperature $T_{c}$ over the critical temperature in the absence of impurities $T_{c_{0}}$ is predicted for a small impurity concentration.
\end{abstract}

Keywords: A. superconductors C. point defects D. phase transitions

Typeset using REVTEX 
Reprinted from Solid State Communications, Vol. 101, No. 1, G. Harań and A. D. S. Nagi, Weak anisotropic impurity scattering in unconventional superconductors, pp. 71-75, 1997, with permission from Elsevier Science Ltd, The Boulevard, Langford Lane, Kidlington 0X5 1GB, UK 
There has been a great interest in studying the properties of unconventional superconductivity in last decade. These superconductors are characterized by order parameter of lower symmetry than the conventional one, which only breaks U(1) gauge symmetry. Such order parameters are involved in almost all theories of heavy fermion and high temperature superconductivity 1 t 4 s the order parameter $\Delta(\mathbf{k})$ is a nontrivial real function of $\mathbf{k}$ its Fermi surface average $\langle\Delta(\mathbf{k})\rangle$ strongly depends on the shape of the Fermi sheet and may take values between -1 and 1 under the normalization condition $\left\langle\Delta^{2}(\mathbf{k})\right\rangle=1$. Most of the theoretical effort has been focussed on the case of $\langle\Delta(\mathbf{k})\rangle=0$ however, and only recently the unconventional superconductivity with a nonvanishing Fermi surface average of the order parameter has been discussed. 11 In the presence of impurities particularly, these superconductors were suggested to show some interesting phenomena in the density of states, which should detectable in the thermodynamic and transport properties. 0 . 99

In this paper, we go beyond the approximation of the isotropic impurity scattering and consider the weak anisotropic (momentum-dependent) impurity potential, which can be treated as a small perturbartion of the isotropic scattering. We show that a really interesting case of weak anisotropic scattering is when the Fermi surface average of the order parameter is nonzero. Particularly for large values of $\langle\Delta(\mathbf{k})\rangle$ we obtain a surprizing increase of the critical temperature $T_{c}$ with the impurity scattering rate in the range of small impurity concentration.

Recent experimental 12.13 as well as theoretical ${ }^{4}$ studies suggest the importance of the anisotropic impurity scattering issue in the interpretation of the $T_{c}$ suppression due to impurities in YBCO. As the anisotropy of the impurity potential is preasumably strong in this compund 3,14 the use of a perturbation method may not be applicable. We believe, however, that the results of the present study may apply to some other anisotropic superconductors like heavy fermions, for instance, where a highly complex Fermi surface 15 (FS) and anisotropic order parameter may lead to a nonzero $\langle\Delta(\mathbf{k})\rangle .16 .17$ We take $\hbar=k_{B}=1$ throughout the paper. 
We assume randomly distributed nonmagnetic impurities in an anisotropic superconductor. Treating the electron-impurity scattering within first-order Born approximation and neglecting the impurity-impurity interaction, 18 the normal and anomalous temperature Green's functions averaged over the impurity positions are given by

$$
\begin{gathered}
G(\omega, \mathbf{k})=-\frac{i \tilde{\omega}+\xi_{k}}{\tilde{\omega}^{2}+\xi_{k}^{2}+|\tilde{\Delta}(\mathbf{k})|^{2}} \\
F(\omega, \mathbf{k})=\frac{\tilde{\Delta}(\mathbf{k})}{\tilde{\omega}^{2}+\xi_{k}^{2}+|\tilde{\Delta}(\mathbf{k})|^{2}}
\end{gathered}
$$

where the renormalized Matsubara frequency $\tilde{\omega}(\mathbf{k})$ and the renormalized order parameter $\tilde{\Delta}(\mathbf{k}) \operatorname{read}$

$$
\begin{gathered}
\tilde{\omega}(\mathbf{k})=\omega+i n_{i} \int\left|w\left(\mathbf{k}-\mathbf{k}^{\prime}\right)\right|^{2} G\left(\omega, \mathbf{k}^{\prime}\right) \frac{d^{3} k^{\prime}}{(2 \pi)^{3}} \\
\tilde{\Delta}(\mathbf{k})=\Delta(\mathbf{k})+n_{i} \int\left|w\left(\mathbf{k}-\mathbf{k}^{\prime}\right)\right|^{2} F\left(\omega, \mathbf{k}^{\prime}\right) \frac{d^{3} k^{\prime}}{(2 \pi)^{3}}
\end{gathered}
$$

In above $\omega=\pi T(2 n+1)$ ( $\mathrm{T}$ is the temperature and $\mathrm{n}$ is an integer number), $\xi_{k}$ is the quasiparticle energy, $n_{i}$ is impurity (defect) concentration, $w\left(\mathbf{k}-\mathbf{k}^{\prime}\right)$ is momentum dependent impurity potential and $\Delta(\mathbf{k})$ is the orbital part of a singlet 19 superconducting order parameter defined as

$$
\Delta(\mathbf{k})=\Delta e(\mathbf{k})
$$

where $e(\mathbf{k})$ is a real basis function of an one dimensional (1D) irreducible representation of a crystal point group or a linear combination of the basis functions of 1D representations. We normalize $e(\mathbf{k})$ by taking $\left\langle e^{2}\right\rangle=1$, where $\langle\ldots\rangle=\int_{F S} d S_{k} n(\mathbf{k})(\ldots)$ denotes the average over the Fermi surface (FS), $n(\mathbf{k})$ is the angle resolved FS density of states normalized to unity, i.e. $\int_{F S} d S_{k} n(\mathbf{k})=1$, and $\int_{F S} d S_{k}$ stands for the integration over the Fermi surface.

The impurity scattering potential is assumed to be separable and given by 


$$
\left|w\left(\mathbf{k}-\mathbf{k}^{\prime}\right)\right|^{2}=|w|^{2} h(\mathbf{k}) h\left(\mathbf{k}^{\prime}\right)
$$

with

$$
h(\mathbf{k})=1+g(\mathbf{k}), \quad|g(\mathbf{k})| \ll 1
$$

which means that $g(\mathbf{k})$ function represents a small anisotropic correction to the isotropic scattering potential. The above functions are normalized by taking $\langle h\rangle=1(\langle g\rangle=0)$. We note from Eq. (3) and the form of impurity potential (Eqs. (6) and (7)) that $\tilde{\omega}$ is $\mathbf{k}$-dependent. This means that the electron self-energy due to impurity scattering and consequently the quasiparticle life-time are anisotropic and change over the Fermi surface. Further, it yields from Eqs. (河), (6), and (7) that the impurity scattering may change the symmetry of the renormalized order parameter $\tilde{\Delta}(\mathbf{k})$ depending on the $g(\mathbf{k})$ symmetry. In that respect our approximation differs from that by Markowitz and Kadanoff 0 as well as that by Millis et al.21 where the authors assumed only a change of a degree of order parameter anisotropy but not the anisotropy function itself.

To proceed further, we restrict the wave vectors of the electron self-energy and pairing potential to the Fermi surface and replace $\int d^{3} k /(2 \pi)^{3}$ by $N_{0} \int_{F S} d S_{k} n(\mathbf{k}) \int d \xi_{k}$, where $N_{0}$ is the overall density of states at the Fermi surface. Using Eqs. (11), (21), (51), and (6) in Eqs. (B) and (4) and performing the integration over $\xi_{k}$ (particle-hole symmetry of quasiparticle spectrum is assumed) we write

$$
\begin{aligned}
& \tilde{\omega}(\mathbf{k})=\omega[1+u(\omega) h(\mathbf{k})] \\
& \tilde{\Delta}(\mathbf{k})=\Delta[e(\mathbf{k})+e(\omega) h(\mathbf{k})]
\end{aligned}
$$

where $u(\omega)$ and $e(\omega)$ functions are determined by the self-consistent equations

$$
\begin{aligned}
& u(\omega)=\Gamma \int_{F S} d S_{k} n(\mathbf{k}) h(\mathbf{k}) \frac{1+u(\omega) h(\mathbf{k})}{\left[\tilde{\omega}^{2}+|\tilde{\Delta}(\mathbf{k})|^{2}\right]^{1 / 2}} \\
& e(\omega)=\Gamma \int_{F S} d S_{k} n(\mathbf{k}) h(\mathbf{k}) \frac{e(\mathbf{k})+e(\omega) h(\mathbf{k})}{\left[\tilde{\omega}^{2}+|\tilde{\Delta}(\mathbf{k})|^{2}\right]^{1 / 2}}
\end{aligned}
$$


and $\Gamma=\pi N_{0} n_{i}|w|^{2}$ is the impurity scattering rate. The gap function is given by the selfconsistent equation

$$
\Delta(\mathbf{k})=-T \sum_{\omega} \sum_{\mathbf{k}^{\prime}} V\left(\mathbf{k}, \mathbf{k}^{\prime}\right) \frac{\tilde{\Delta}\left(\mathbf{k}^{\prime}\right)}{\tilde{\omega}^{2}+\xi_{k^{\prime}}^{2}+\left|\tilde{\Delta}\left(\mathbf{k}^{\prime}\right)\right|^{2}}
$$

where $V\left(\mathbf{k}, \mathbf{k}^{\prime}\right)$ is the phenomenological pair potential taken as

$$
V\left(\mathbf{k}, \mathbf{k}^{\prime}\right)=-V_{0} e(\mathbf{k}) e\left(\mathbf{k}^{\prime}\right)
$$

Following standard procedure,22 we obtain the equation for the critical temperature $T_{c}$ as

$$
\ln \frac{T_{c}}{T_{c_{0}}}=2 \pi T_{c} \sum_{\omega>0}\left[(f(\omega))_{\Delta=0}-\frac{1}{\omega}\right]
$$

with

$$
(f(\omega))_{\Delta=0}=\int_{F S} d S_{k} n(\mathbf{k}) \frac{e(\mathbf{k})}{\tilde{\omega}_{0}(\mathbf{k})}\left[\frac{\tilde{\Delta}(\mathbf{k})}{\Delta}\right]_{\Delta=0}
$$

where $T_{c_{0}}$ is the critical temperature in the absence of impurities and $\tilde{\omega}_{0}(\mathbf{k})=\tilde{\omega}(\mathbf{k})_{\Delta=0}$. Using Eqs. (7)-(11) and neglecting terms of order $g^{2}(\mathbf{k})$ and higher, we obtain for $\Delta \rightarrow 0$

$$
\begin{gathered}
\tilde{\omega}_{0}(\mathbf{k})=\omega+\Gamma(1+g(\mathbf{k})) \operatorname{sign}(\omega) \\
{\left[\frac{\tilde{\Delta}(\mathbf{k})}{\Delta}\right]_{\Delta=0}=e(\mathbf{k})+\left(\langle e\rangle+\langle e g\rangle\left(1-\frac{\Gamma}{|\omega|+\Gamma}\right)\right) \frac{\Gamma}{|\omega|}[1+g(\mathbf{k})]}
\end{gathered}
$$

We note from Eq. (17) that the impurity scattering induces anisotropy in the renormalized order parameter $\tilde{\Delta}(\mathbf{k})$ other than that of $\Delta(\mathbf{k})$ which is described by a function $e(\mathbf{k})$ (Eq. (5)). There are two different sources of this additional anisotropy in $\tilde{\Delta}(\mathbf{k})$. First of them is a nonzero value of $\langle e\rangle$ which is determined by the symmetry of the order parameter and that of the Fermi surface, and the second reflected in Eq. (17) by $g(\mathbf{k})$ function and coupling coefficient $\langle e g\rangle$ is the anisotropy of the impurity scattering potential.

Based on Eqs. (15), (16), and (17) we obtain from Eq. (14) 


$$
\begin{aligned}
& \ln \frac{T_{c}}{T_{c_{0}}}=\left(\langle e\rangle^{2}-1\right)\left(\psi\left(\frac{1}{2}+\frac{\Gamma}{2 \pi T_{c}}\right)-\psi\left(\frac{1}{2}\right)\right)+ \\
& \left(2\langle e\rangle\langle e g\rangle-\left\langle e^{2} g\right\rangle\right) \frac{\Gamma}{2 \pi T_{c}} \psi^{(1)}\left(\frac{1}{2}+\frac{\Gamma}{2 \pi T_{c}}\right)
\end{aligned}
$$

where $\psi(z)$ and $\psi^{(1)}(z)$ are digamma and trigamma functions respectively. The first term on the righthand side of Eq. (18) represents the isotropic impurity scattering effect on $T_{c} 2325$ and the influence of the weak anisotropic scattering is reflected by the second term. Compared to the isotropic pair-breaking, the weak anisotropic impurity potential can lead either to a small (of order $g$ ) additional decrease of $T_{c}$ when the term $\left(2\langle e\rangle\langle e g\rangle-\left\langle e^{2} g\right\rangle\right)$ is negative or to a small reduction of $T_{c}$ suppression for a positive value of $\left(2\langle e\rangle\langle e g\rangle-\left\langle e^{2} g\right\rangle\right)$.

We consider the case of a positive value of this parameter in more detail. For the sake of convenience we assume $\left\langle e^{2} g\right\rangle=0$ and then deal with the order parameter-impurity potential coupling coefficient $\langle e g\rangle$, which does not reduce the generality of approach but simplifies the notation. One would obtain the same results without the above assumption using the term $\left(2\langle e\rangle\langle e g\rangle-\left\langle e^{2} g\right\rangle\right)$ as a parameter with the values assigned to $2\langle e\rangle\langle e g\rangle$ coefficient in this paper. The actual value of $\left\langle e^{2} g\right\rangle$ depends on two factors: the symmetry of both $e(\mathbf{k}), g(\mathbf{k})$ functions and the shape of the Fermi surface. Thus the estimation of $\left\langle e^{2} g\right\rangle$ parameter is difficult in the real systems, however, its value may vanish in some cases (see Appendix).

We present the critical temperature $T_{c}$ normalized by the critical temperature in the absence of impurities $T_{c_{0}}$ as a function of normalized impurity scattering rate $\Gamma / 2 \pi T_{c_{0}}$ for different values of the impurity coupling coefficient $\langle e g\rangle$ in Figs. 1-3. As we are considering a weak anisotropic potential and have neglected terms of order $\langle e g\rangle^{2}$ while writing Eq. (18), we take values of $\langle e g\rangle$ upto $\sim 0.3$. We notice from these figures that the effect of anisotropic scattering is very weak for small Fermi surface average values of the order parameter $\langle e\rangle$ and may not be distinguished from the isotropic impurity scattering ( Figs. 1-2), nevertheless the influence of the anisotropy in the scattering potential increases with the increase in $\langle e\rangle$. For the large values of the Fermi surface averaged order parameter that is $\langle e\rangle \sim 0.9$ (Fig. 3) and for the coupling coefficient $\langle e g\rangle$ larger than approximately 0.1 we observe a very 
interesting feature of an initial enhancement of $T_{c}$ for small impurity concentration. This initial enhancement of $T_{c}$ over $T_{c_{0}}$ may be understood by writing Eq. (18) for small impurity concentration (i.e. $\Gamma / 2 \pi T_{c_{0}} \ll 1$ ). We have in that case

$$
\frac{T_{c}}{T_{c_{0}}}-1 \simeq \frac{\pi^{2} \alpha}{2}\left(\frac{\Gamma}{2 \pi T_{c_{0}}}\right)
$$

where $\alpha=\langle e\rangle^{2}-1+2\langle e\rangle\langle e g\rangle-\left\langle e^{2} g\right\rangle$. Therefore $T_{c}$ is an increasing function of $\Gamma$ for a positive $\alpha$ and decreases with the impurity concentration for a negative $\alpha$. This criterion may serve for estimation of $\langle e g\rangle$ value which gives rise to the enhancement of the critical temperature with scattering rate. For example for $\langle e\rangle=0.8\left(\left\langle e^{2} g\right\rangle=0\right)$ this coefficient is $\langle e g\rangle \sim 0.23$, and for $\langle e\rangle=0.9\left(\left\langle e^{2} g\right\rangle=0\right)$ the coefficient $\langle e g\rangle=0.2$ leads to the $T_{c}$ enhancement of about $10 \%$ for $\Gamma / 2 \pi T_{c_{0}} \simeq 0.12$ (as in Fig. 3). The required large $\langle e\rangle$ value suggests a substantial s-wave component in the order parameter.

Although in above we have taken $\left\langle e^{2} g\right\rangle=0$, we expect, that even when $\left\langle e^{2} g\right\rangle$ coefficient has a positive value, the factor $\alpha$ can be made positive by appropriately large value of $\langle e\rangle$. It is worth mentioning that the above results cannot be obtained with the assumption of the proportionality of the impurity potential anisotropy function $g(\mathbf{k})$ to the order parameter orbital function $e(\mathbf{k})$, since the constraint $\langle g\rangle=0$ would yield $\langle e\rangle=0$.

In summary, we have investigated the effect of weak anisotropic impurity scattering in unconventional superconductors. It is shown that the anisotropy of the impurity potential can lead to either a small increase or a small decrease in $T_{c}$ suppression due to isotropic scattering. The influence of weak anisotropic scattering becomes significant for the order parameters with large Fermi surface average values $\langle e\rangle$. In this case the critical temperature is increased up to 10 per cent over $T_{c_{0}}$ for small impurity concentration. This anisotropic scattering-induced $T_{c}$ enhancement in unconventional superconductors with large $\langle e\rangle$ values is a novel feature of the present study.

This work was supported by the Natural Sciences and Engineering Research Council of 
Canada.

\section{APPENDIX:}

We assume $e(\mathbf{k})$ and $g(\mathbf{k})$ as the basis functions of 1D irreducible representations of the crystal point group. Therefore for a symmetry operation from this group $S_{i}$

$$
S_{i} g(\mathbf{k})=g_{i} g(\mathbf{k})
$$

where $g_{i}$ is a number. Further, if there is a part $P$ of FS which reproduces the whole Fermi sheet under the crystal group symmetry operations $\left(\mathrm{FS}=\sum_{i} S_{i} P\right)$ and the average value of $g(\mathbf{k})$ over this segment of FS does not vanish, i.e. $\langle g\rangle_{P} \neq 0$, then from the normalization $\langle g\rangle=0$ and from the relation

$$
\langle g\rangle=\sum_{i}\left\langle S_{i} g\right\rangle_{P}=\langle g\rangle_{P} \sum_{i} g_{i}
$$

we get

$$
\sum_{i} g_{i}=0
$$

Taking into account that $e^{2}(\mathbf{k})$ is an identity representation for a real $e(\mathbf{k})$, which means that $S_{i} e^{2}(\mathbf{k})=e^{2}(\mathbf{k})$, we calculate $\left\langle e^{2} g\right\rangle$ as follows

$$
\left\langle e^{2} g\right\rangle=\sum_{i}\left\langle S_{i}\left(e^{2} g\right)\right\rangle_{P}=\left\langle e^{2} g\right\rangle_{P} \sum_{i} g_{i}
$$

Then Eqs. (A3) and (A4) yield $\left\langle e^{2} g\right\rangle=0$.

On the other hand, if the order parameter is given by a linear combination of a s-wave component and a basis function $f(\mathbf{k})$ of a nonidentity 1D irreducible representation of the crystal point group, that is

$$
e(\mathbf{k})=\langle e\rangle+f(\mathbf{k})
$$

then $\langle e g\rangle=\langle f g\rangle$ and under the same assumptions about $g(\mathbf{k})$ we get $\left\langle e^{2} g\right\rangle=2\langle e\rangle\langle e g\rangle$ which leads to a cancelation of the anisotropy of the scattering potential in $T_{c}$ equation 
(Eq. (18)). We conclude from this, that in order to observe the effect of weak anisotropic scattering, $e(\mathbf{k})$ and $g(\mathbf{k})$ must belong to $A_{1 g}$ representation with $g(\mathbf{k})$ normalized to fulfill $\langle g\rangle=0$.

The above considerations, however, are based on the assumption that $g(\mathbf{k})$ transforms according to the symmetry of the crystal point group and are not valid in the case of $g(\mathbf{k})$ described by a symmetry other than that of the crystal lattice. 


\section{REFERENCES}

* on leave from: Institute of Physics, Politechnika Wrocławska, Wybrzeże Wyspiańskiego 27, 50-370 Wrocław, Poland

${ }^{1}$ M. Sigrist and K. Ueda, Rev. Mod. Phys. 63 (1991) 239

${ }^{2}$ J. Annett, N. Goldenfeld and A. J. Leggett, in Physical Properties of High Temperature Superconductors, Vol. 5, D. M. Ginsberg (ed.), (World Scientific, Singapore, 1996)

${ }^{3}$ D. Pines and P. Monthoux, J. Phys. Chem. Solids 56, 1651 (1995)

${ }^{4}$ D. J. Scalapino, Phys. Reports 250, 329 (1995)

${ }^{5}$ L. S. Borkowski and P. J. Hirschfeld, Phys. Rev. B49, 15404 (1994)

${ }^{6}$ L. S. Borkowski, P. J. Hirschfeld, and W. O. Putikka, Phys. Rev. B52, 3856 (1995)

${ }^{7}$ R. Fehrenbacher and M. R. Norman, Physica C 235-240, 2407 (1994); R. Fehrenbacher and M. R. Norman, Phys. Rev. B50, 3495 (1994)

${ }^{8}$ C. O’Donovan, D. Branch, J. P. Carbotte, and J. S. Preston, Phys. Rev. B51, 6588 (1995);

C. O’Donovan and J. P. Carbotte, Phys. Rev. B52, 4568 (1995)

${ }^{9}$ H. Kim and E. J. Nicol, Phys. Rev. B52, 13576 (1995)

${ }^{10}$ S. V. Pokrovsky and V. L. Pokrovsky, Phys. Rev. Lett. 75, 1150 (1995)

${ }^{11}$ G. Preosti and P. Muzikar, preprint

12 A. G. Sun, L. M. Paulius, D. A. Gajewski, M. B. Maple and R. C. Dynes, Phys. Rev. B50, 3266 (1994); J. M. Valles, Jr., A. E. White, K. T. Short, R. C. Dynes, J. P. Garno, A. F. Levi, M. Anzlowar and K. Baldwin, Phys. Rev. B39, 11599 (1989)

13 J. Giapintzakis, D. M. Ginsberg, M. A. Kirk and S. Ockers, Phys. Rev. B50, 15967 (1994)

${ }^{14}$ G. Harań and A. D. S. Nagi, Phys. Rev. B54 (1996) 
15 T. Oguchi and A. J. Freeman, J. Magn. Magn. Mater. 61, 233 (1986); M. R. Norman, R. C. Albers, A. M. Boring and N. E. Christensen, Solid State Commun. 68, 245 (1988)

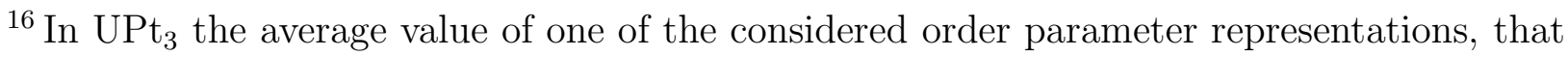
is d-wave $A_{1 g}$ with $\Delta(\mathbf{k})=\Delta\left(2 k_{z}^{2}-k_{x}^{2}-k_{y}^{2}\right)$, taken over two sheets of FS approximated as in Ref. 14 is $\langle\Delta(\mathbf{k})\rangle \simeq 0.1 \Delta$.

${ }^{17}$ G. Harań, P. J. Hirschfeld and M. Sigrist, preprint

18 A. A. Abrikosov and L. P. Gorkov, Zh. Eksp. Teor. Fiz. 39, 1781 (1960) [Sov. Phys. JETP 12, 1243 (1961)]; see also A. A. Abrikosov, L. P. Gorkov,and I. E. Dzyaloshinski, Methods of Quantum Field Theory in Statistical Physics (Dover, New York, 1975), sec 39

${ }^{19}$ Our discussion is also valid for a triplet-paired state since the nonmagnetic impurities do not affect the spin part of the order parameter.

${ }^{20}$ D. Markowitz and L. P. Kadanoff, Phys. Rev. 131, 563 (1963)

${ }^{21}$ A. J. Millis, S. Sachdev and C. M. Varma, Phys. Rev. B37, 4975 (1988)

${ }^{22}$ K. Maki, in Superconductivity, R. D. Parks (ed.), (Marcel Dekker, New York, 1969), Vol. 2, pp. $1035-1102$

${ }^{23}$ A. A. Abrikosov, Physica C 214, 107 (1993)

${ }^{24}$ R. J. Radtke, K. Levin, H.-B. Schüttler and M. R. Norman, Phys. Rev. B48, 653 (1993)

${ }^{25}$ S. K. Tolpygo, J.-Y. Lin, M. Gurvitch, S. Y. Hou and J. M. Phillips, Phys. Rev. B53, 12454 (1996); S. K. Tolpygo, J.-Y. Lin, M. Gurvitch, S. Y. Hou and J. M. Phillips, Phys. Rev. B53, 12462 (1996) 


\section{FIGURES}

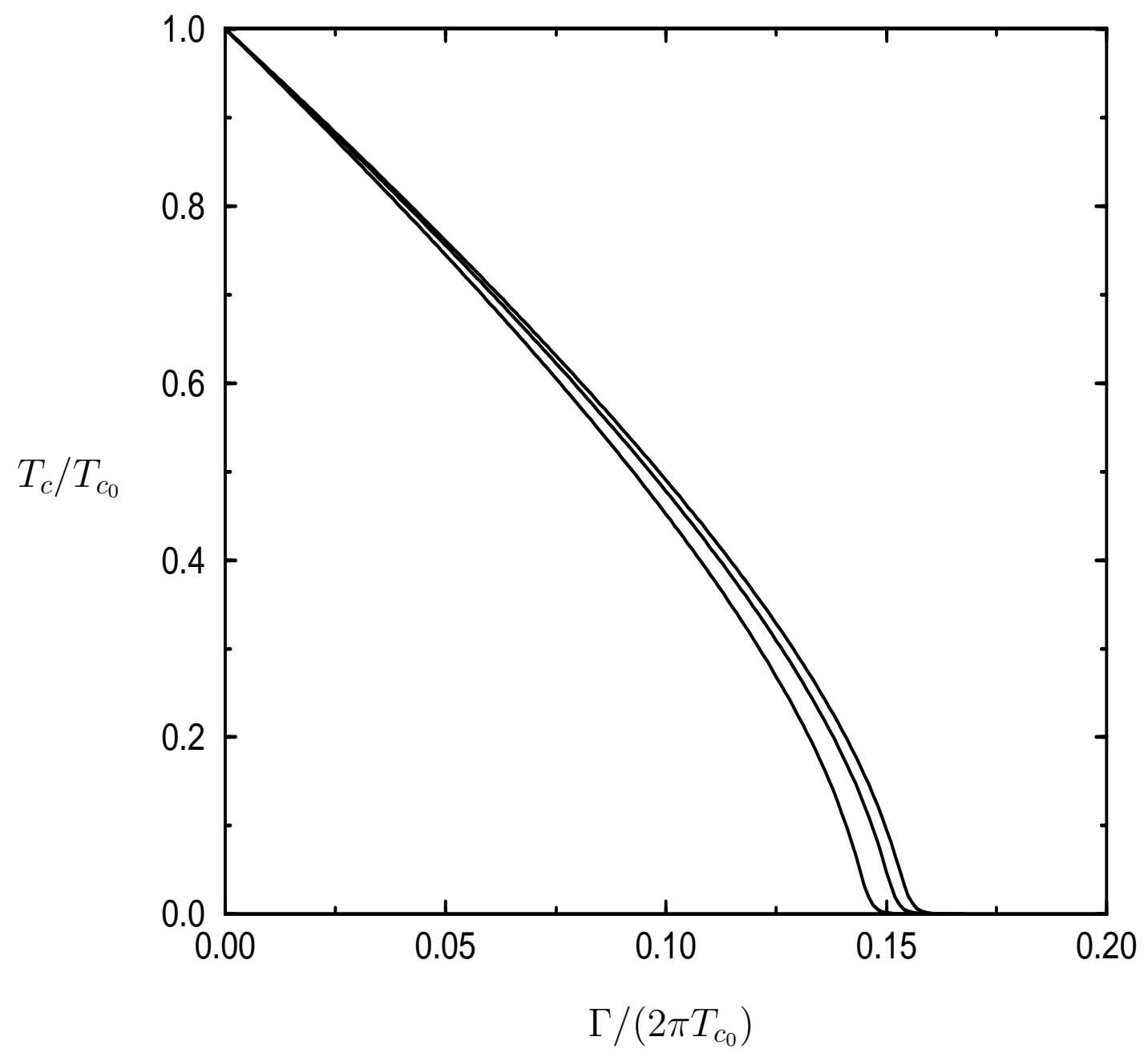

FIG. 1. Normalized critical temperature $T_{c} / T_{c_{0}}$ as a function of the normalized scattering rate $\Gamma / 2 \pi T_{c_{0}}$ for $\langle e\rangle=0.1$. From the bottom to the top, curves are plotted for $\langle e g\rangle=0$ (isotropic scattering), 0.2 , and $0.3 ;\left\langle e^{2} g\right\rangle=0$ is assumed. 


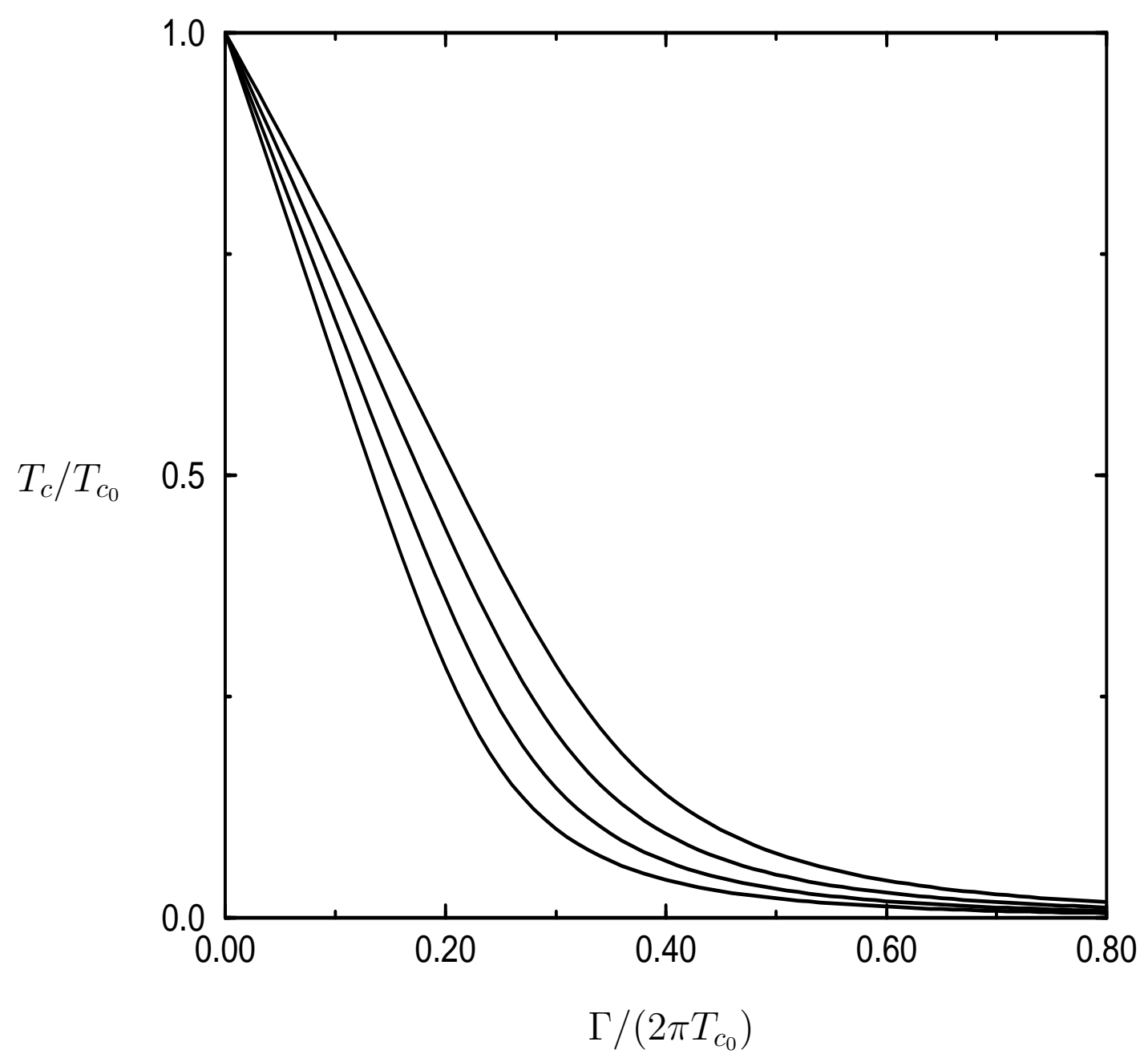

FIG. 2. Normalized critical temperature $T_{c} / T_{c_{0}}$ as a function of the normalized scattering rate $\Gamma / 2 \pi T_{c_{0}}$ for $\langle e\rangle=0.5$. From the bottom to the top, curves are plotted for $\langle e g\rangle=0$ (isotropic scattering), $0.1,0.2$, and $0.3 ;\left\langle e^{2} g\right\rangle=0$ is assumed. 


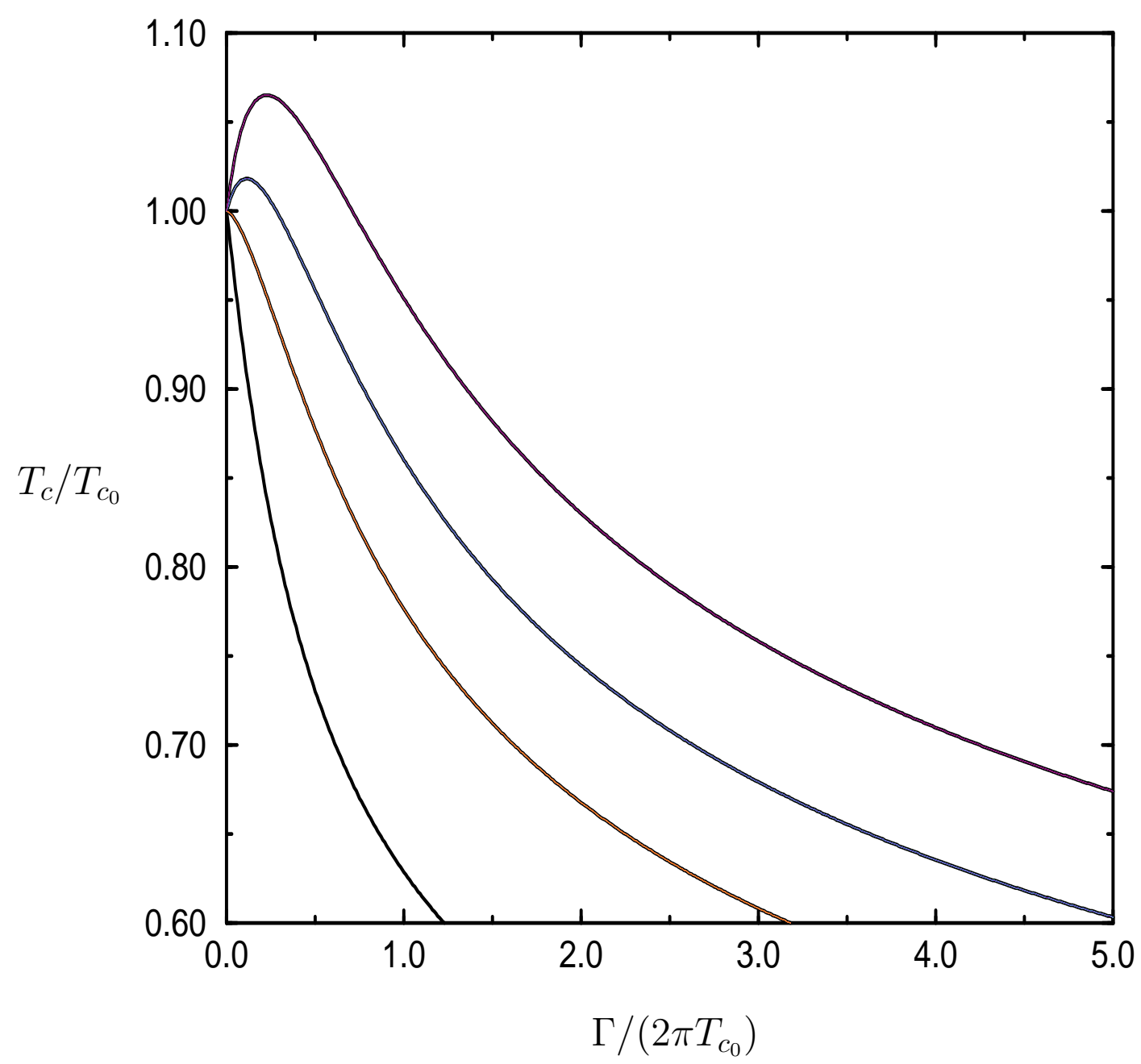

FIG. 3. Normalized critical temperature $T_{c} / T_{c_{0}}$ as a function of the normalized scattering rate $\Gamma / 2 \pi T_{c_{0}}$ for $\langle e\rangle=0.9$. From the bottom to the top, curves are plotted for $\langle e g\rangle=0$ (isotropic scattering), 0.1, 0.15, and $0.2 ;\left\langle e^{2} g\right\rangle=0$ is assumed. 